OPEN ACCESS

Edited by:

Basem M. William,

The Ohio State University

United States

Reviewed by:

Apostolos Zaravinos,

European University Cyprus, Cyprus

Zhiming $L i$,

Sun Yat-sen University Cancer Center

(SYSUCC), China

*Correspondence:

Xiangdong $L$

xiangdongli@cau.edu.cn

Specialty section: This article was submitted to Hematologic Malignancies, a section of the journal

Frontiers in Oncology

Received: 02 April 2019 Accepted: 10 February 2020

Published: 05 March 2020

Citation:

Liu H, Liu M, You H, LiX and LiX (2020) Oncogenic Network and Hub Genes for Natural Killer/T-Cell Lymphoma Utilizing WGCNA.

Front. Oncol. 10:223

doi: 10.3389/fonc.2020.00223

\section{Oncogenic Network and Hub Genes for Natural Killer/T-Cell Lymphoma Utilizing WGCNA}

\author{
Huijiao Liu ${ }^{1,2}$, Mei Liu ${ }^{3}$, Hua You ${ }^{2}$, Xiru $\mathrm{Li}^{4}$ and Xiangdong $\mathrm{Li}^{1,2,5 *}$ \\ ${ }^{1}$ Beijing Advanced Innovation Center for Food Nutrition and Human Health, College of Biological Sciences, China Agricultural \\ University, Beijing, China, ${ }^{2}$ Affiliated Cancer Hospital \& Institute of Guangzhou Medical University, Guangzhou, China, \\ ${ }^{3}$ Department of Pathology, General Hospital of PLA, Beijing, China, ${ }^{4}$ Department of General Surgery, The 301th Hospital of \\ PLA, Beijing, China, ${ }^{5}$ State Key Laboratory of Agrobiotechnology, College of Biological Sciences, China Agricultural \\ University, Beijing, China
}

Natural killer (NK)/T-cell lymphoma (NKTCL) is a subtype of non-Hodgkin lymphoma with aggressive progression and poor prognosis. The molecular mechanisms of NKTCL have not been well-studied. Herein, we revealed the lymphoma-associated dysregulated genes and signaling pathways or biological processes in NKTCL. We characterized that the extracellular matrix (ECM) receptor interaction pathway and T-cell receptor signaling pathway were the main dysregulated pathways in NKTCL by Gene Ontology (GO) analysis and pathway enrichment analysis. By using weighted gene co-expression network analysis (WGCNA), the gene co-expression network of NKTCL (SRP049695) was constructed, and hub genes (LMO3, GRB14) were identified. In addition, another Gene Expression Omnibus (GEO) dataset (GSE69406) was used to validate these hub genes. Furthermore, these hub genes were identified and validated by survival analysis (GSE90597). These results provided novel insights into the pathogenesis of NKTCL. Of particular interest, LMO3 and GRB14 might be potential oncoproteins and biomarkers for the diagnosis and treatment of NKTCL.

Keywords: natural killer/T-cell lymphoma (NKTCL), weight gene co-expression network analysis (WGCNA), co-expression network, LMO3, GRB14

\section{INTRODUCTION}

Natural killer (NK)/T-cell lymphoma (NKTCL) is a rare but aggressive lymphoid malignancy with poor prognosis and could be subdivided into two subtypes: nasal type and extra-nasal type (1). Still, NKTCL does not have a standard classification and treatment. Five years of overall survival (OS) of nasal type and extra-nasal type are 42 and 9\%, respectively (2). So far, the pathogenesis of NKTCL remains unclear.

Recent works have achieved some progress in NKTCL by using array-based technologies. Somatic alterations, including TP53, DDX3X, and STAT3 mutations, have been identified in $\operatorname{NKTCL}(3,4)$. TP53 functions as a tumor suppressor gene, which mainly leads cell cycle to arrest at the G1 phase. TP53 mutations are found in $20-60 \%$ of NKTCL $(5,6)$. DDX3X is an ATP-dependent RNA helicase and plays an important role in the nucleus by regulating transcription. A larger-scale research revealed a high frequent alteration (20\%) of DDX3X in NKTCL (3). STAT3 and STAT5B were found mutated in about $12 \%$ frequency (7). Some single genes, such as surviving, Aurora Kinase A (AURKA), C-MYC, EZH2, RUNX3, were found to be deregulated in NKTCL (8-12). 
Several studies have provided evidence that the Janus kinase (JAK)/signal transducer and activator of transcription (STAT), platelet-derived growth factor (PDGF), NOTCH-1, nuclear factor $(\mathrm{NF})-\kappa \mathrm{B}$ pathways in NKTCL were dysregulated $(9,13,14)$. Genes involved in core co-expression network in NKTCL still need to be studied.

In previous work, most researchers focused on the screening of the most differential genes. Weighted gene co-expression network analysis (WGCNA) is widely used to explore the huge and complex relationships among genes across microarray or RNA sequence data (15). WGCNA provides a convenient and effective solution for screening core genes that could be potential biomarkers for clinical prognosis and treatment $(16,17)$.

In our study, WGCNA was first used to analyze the hub genes of NKTCL samples mined from the SRA database. Two other GEO databases were used to validate the results. Our findings may provide a new idea of the basic research of NKTCL and beneficial to the clinical diagnosis and treatment.

\section{MATERIALS AND METHODS}

\section{Data Collection and Preprocessing}

Three normal NK normal control and 15 NKTCL case (Supplementary Table 1) RNA-Seq raw data files were downloaded from the National Center for Biotechnology Information (NCBI) Sequence Read Archive (SRA) with the ID SRP049695. TrimGalore (http://www.bioinformatics. babraham.ac.uk/projects/trim_galore/) method was used to filter low-quality raw reads as follows: (1) removal of primers sequencing; (2) removal of sequences of low quality in terminal; (3) removal of sequences $<35 \mathrm{bp}$. Clean reads were aligned with HISAT2 to the USCS hg19 human genome assembly. mRNA and lncRNA quantitative expressions [fragments per kilobase million (FPKM)] were performed by Cufflinks (http://cole-trapnell-lab. github.io/cufflinks).

\section{Differentially Expressed Genes Screening}

The Cuffdiff was used to screen the differentially expressed genes (DEGs) between normal NK cells and NKTCL cases. The DEGs threshold was set as follows: (1) $p<0.05$; (2) $\log _{2}$ (fold change) $>1$ or $\log _{2}$ (fold change) $<-1$; (3) $q<0.05$. ggplot2 package in $\mathrm{R}$ was used to show the heat map and volcano maps.

\section{Gene Ontology Annotations and Kyoto Encyclopedia of Genes and Genomes Pathway Analyses}

Gene Ontology (GO) analysis was performed to show the unique biological significance based on differentially expressed genes. The Kyoto Encyclopedia of Genes and Genomes (KEGG) database was carried out to find out the important pathway. The ClusterProfiler packages (18) in $\mathrm{R}$ was applied to analysis and demonstrated GO annotations and KEGG pathway.

\section{Gene Set Enrichment Analysis}

Expression dataset from SRP049695 was converted to the tabdelimited GCT format as follows: The first column of the GCT file contains gene symbols. The second column was filled with "NA." Subsequent columns were filled with each sample's expression

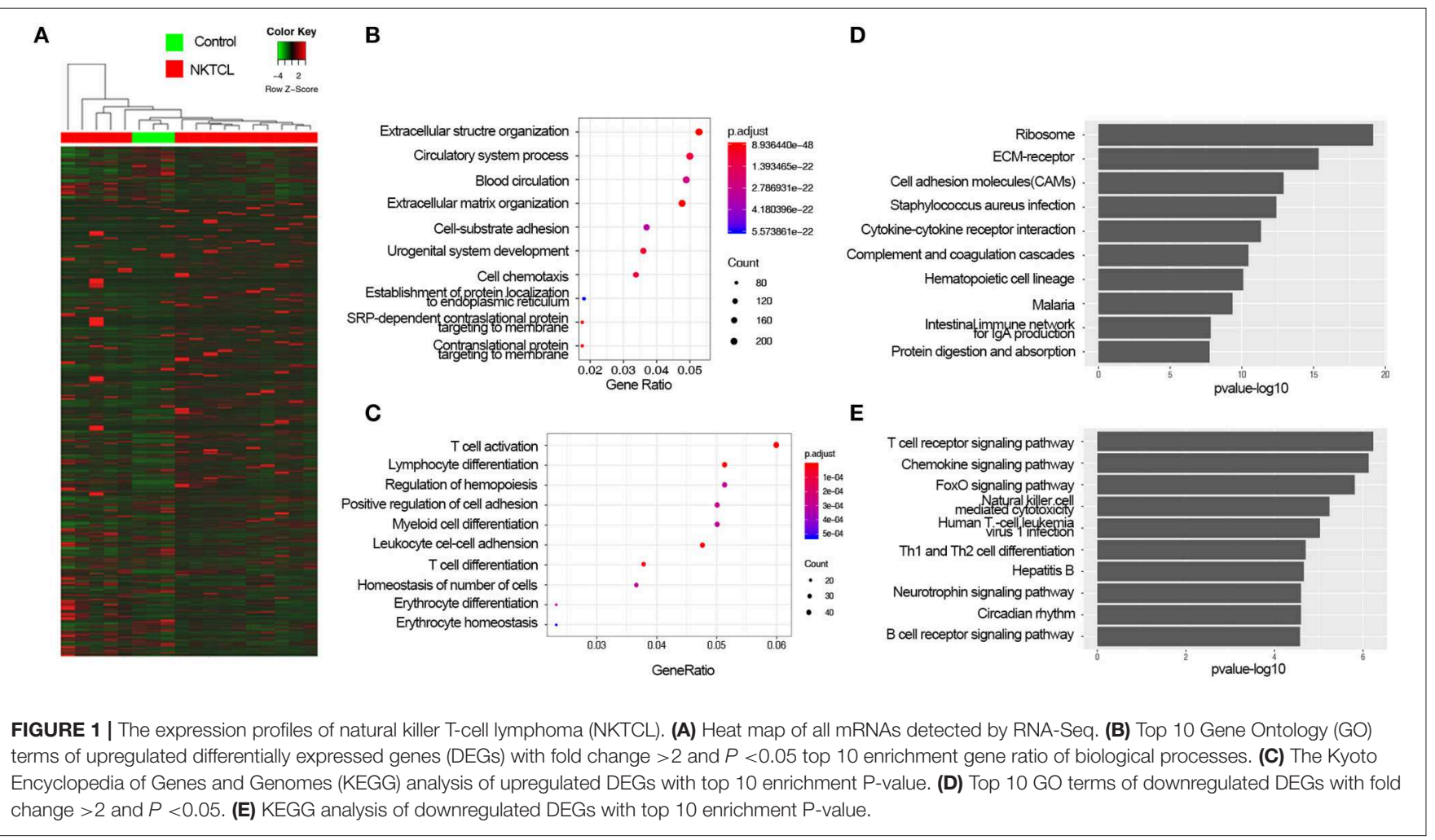


value. The following operations were carried out according to the protocol (http://www.gsea-msigdb.org/gsea/) $(19,20)$.

\section{Protein-Protein Interaction Network Building}

Differentially expressed mRNAs (fold change $>2$ or fold change $<0.5, P<0.05)$ were taken into the Search Tool for the Retrieval of Interacting Genes/Proteins (STRING). The confidence score was set at 0.9. The Molecular Complex Detection (MCODE) was used to analyze the core modules of the protein-protein interaction (PPI) network.

\section{Co-expression Network Construction}

WGCNA package of $\mathrm{R}$ software was applied to uncover the correlation among genes. Firstly, expression data of DEGs was input into $\mathrm{R}$ software to inspect good genes and samples, SRR1648324 and SRR1648323 were excluded from the analysis due to the poor quality. The power of $\beta$ was set at 14 to ensure a scale-free network. The minimum number of module genes was set at 30 . The hierarchical clustering dendrogram summarized the Gene modules with different colors. Heat map and topological overlap matrix (TOM) plot were used to visualize the module structure. The threshold of output to Cytoscape was set at 0.6 .

\section{Hub Gene Selection and Validation}

Gene network files exported from WGCNA analysis were input into Cytoscape software. The MCODE plugin of Cytoscape was used to calculate K-core value of each gene. GSE69406 dataset was used to validate the expression of the hub genes. GSE90597 dataset was used to plot the Kaplan-Meier survival curve in ggplot2 of R software.

\section{RESULTS}

\section{Overview of the Transcriptomes of Natural Killer/T-Cell Lymphoma}

In order to elucidate the molecular pathogenesis of NKTCL, heat maps of all mRNAs were shown in Figure 1A. To assess differential gene expressions between NKTCL and

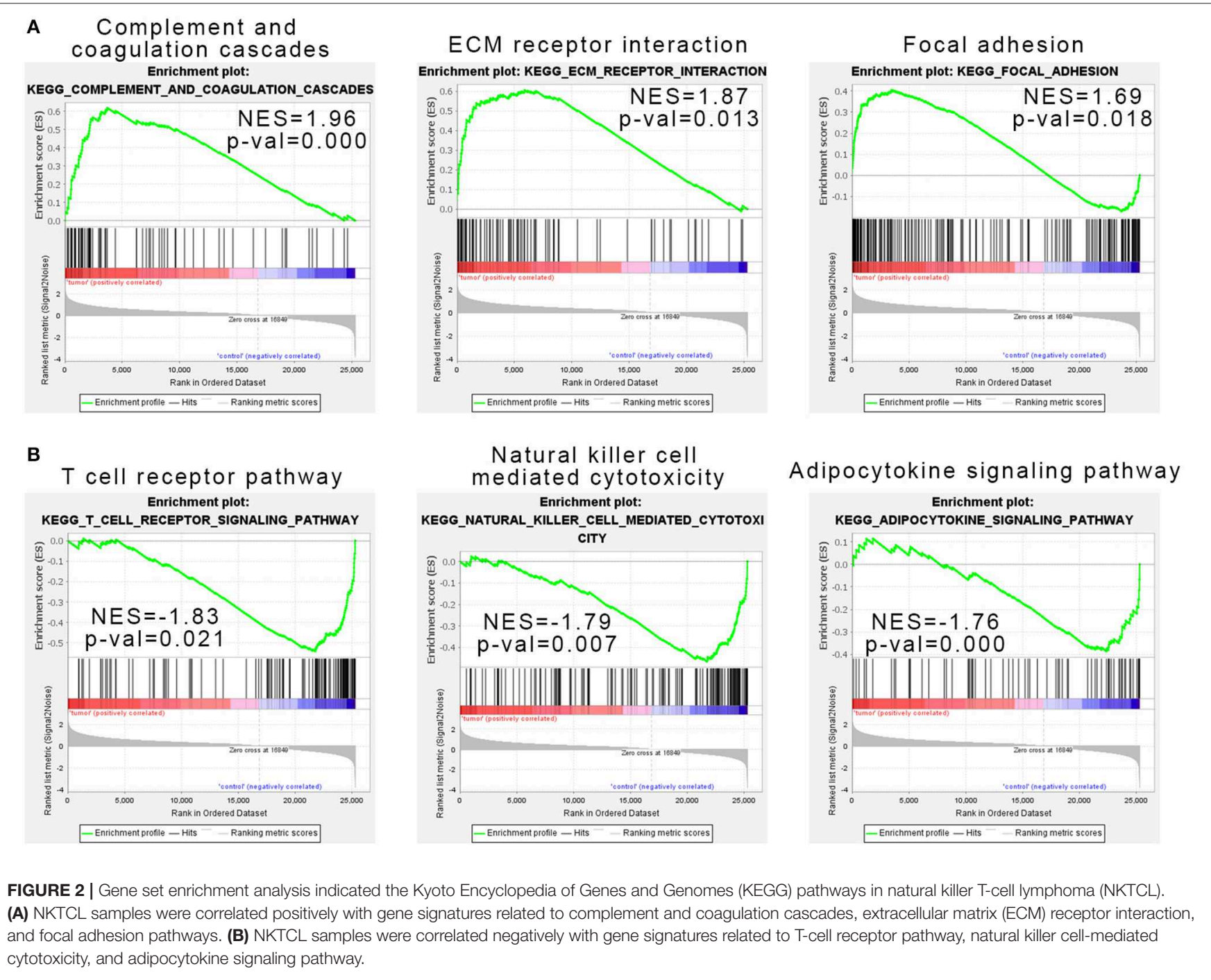


the normal NK cells, all genes were plotted in volcano plots. In total, 6,680 mRNAs displayed the differential expressions in NKTCL, including 5,664 upregulated and 1,016 downregulated mRNAs (Supplementary Figure 1A). Of note, 6,005 lncRNAs showed differential expressions, 4,968 lncRNAs were upregulated, and 1,037 lncRNAs were downregulated (Supplementary Figure 1B). Supplementary Figures 1C,D showed the dysregulated mRNAs or lncRNAs that were $\log _{2}$ Foldchange $\mid>10$ in NKTCL.

\section{Gene Ontology and Kyoto Encyclopedia of Genes and Genomes Analysis of the Differentially Expressed Genes}

To get an insight into the function of DEGs of NKTCL, the upregulated and downregulated DEGs were analyzed by enrichGO or enrichKEGG function of clusterProfiler packages in $\mathrm{R}$ software, respectively. GO analysis results were enriched in the biological process (BP), the upregulated DEGs significantly enriched in the extracellular structure organization, circulatory system process, blood circulation, extracellular matrix (ECM) organization (Figure 1B), and the downregulated DEGs significantly enriched in T-cell activation, lymphocyte differentiation, regulation of hemopoiesis, positive regulation of cell adhesion, myeloid cell differentiation, and leukocyte cell-cell adhesion (Figure 1C). KEGG analysis showed that the upregulated DEGs significantly were enriched in ribosome, ECM-receptor interaction, and cell adhesion molecules (CAMs) pathways (Figure 1D), and the downregulated DEGs were enriched in T-cell receptor signaling pathway, chemokine signaling pathway, and FoxO signaling pathway (Figure 1E). To illustrate the interactions of the upregulated and downregulated pathways in the progression of NKTCL, the top 10 of upregulated and downregulated enriched pathways and their genes were used to construct a network. The T-cell receptor signaling pathway and CAMs pathway were considered to be at the central network, ECM-receptor interaction pathway was found to be the linker between the upregulated pathways and the downregulated pathways (Supplementary Figure 2A). In order to further understand the changes of the pathogenesis in NKTCL, all DEGs, including the upregulated and downregulated genes, were put into KEGG pathway analysis, the molecular network constructed by top 20 of enriched pathways demonstrated that the phosphoinositide 3-kinase (PI3K)/Akt pathway is the most important signal in NKTCL (Supplementary Figure 2B).

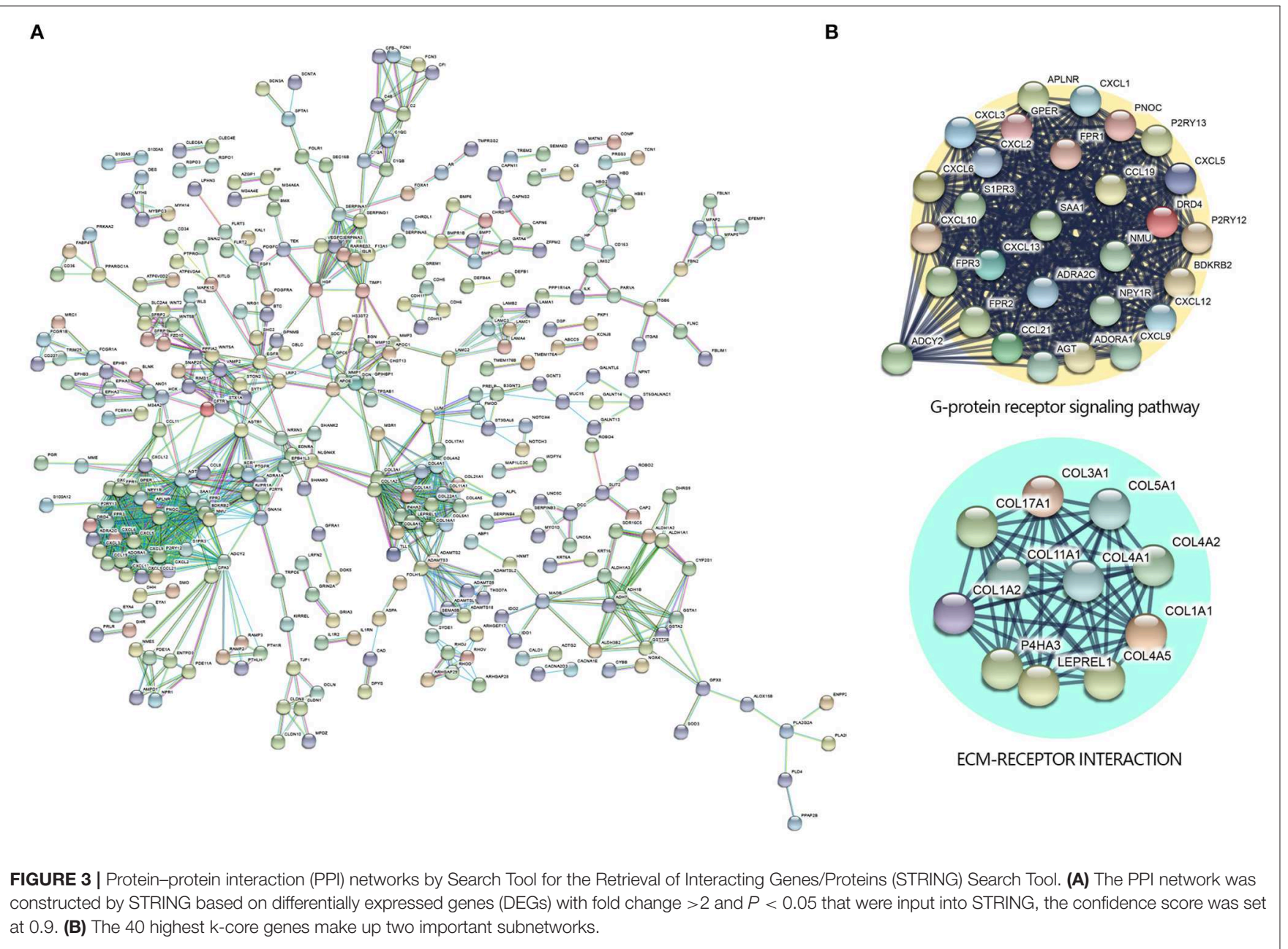




\section{Gene Set Enrichment Analysis of Natural Killer/T-Cell Lymphoma}

Considering that the number of genes input in GO and KEGG analysis is our artificially defined standard $(p<0.05$ and $\mid \log _{2}$ (FC) $\mid>1$ ), the results may be different under different standards (such as $p<0.01$ and $\left|\log _{2}(\mathrm{FC})\right|>1$ ). Here we used all expression data sets of SRP049695 for Gene Set Enrichment Analysis (GSEA) analysis, and the results show that complement and coagulation cascades, ECM receptor interaction, and focal adhesion pathways were enriched in the tumor samples (Figure 2A). T-cell receptor pathway, NK cellmediated cytotoxicity, and adipocytokine signaling pathway were enriched in the control samples (Figure 2B).

\section{Protein-Protein Interaction of Natural Killer T-Cell Lymphoma}

To further investigate the DEGs and the potential protein levels, the STRING database was applied for revealing the core PPI network. As shown in Figure 3A, 982 nodes and 1,076 edges constructed the core PPI network. The network data were reanalyzed by MCODE to get the k-core which reflected the importance of each gene. The 40 highest $\mathrm{k}$-core genes make up two important networks, one is the G protein-coupled receptor signaling pathway, the other is ECM receptor signaling pathway (Figure 3B), which may suggest the underlying mechanism in the NKTCL pathogenesis.

\section{Weighted Gene Correlation Network Analysis of Natural Killer T-Cell Lymphoma}

To clarify the key modules and genes in NKTCL, WGCNA was used to uncover the highly correlated genes and the coexpression networks of NKTCL. SRR1648323 and SRR1638324 samples were excluded from analysis after quality assessment (Figure 4A). The power of $\beta$ was automatically set at 14 to ensure a scale-free network (Figure 4B). Gene modules were calculated, and the gray module represents genes that cannot be clustered into any other modules (Figure 4C). Finally, 18 gene modules were identified by the hierarchical clustering dendrogram. The interactions between gene modules were subsequently analyzed, and the TOM plot of a gene network was generated with the corresponding hierarchical clustering dendrogram and the modules (Figure 4D).

\section{A}

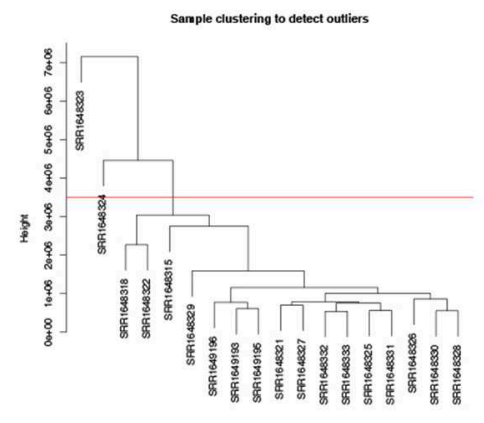

C

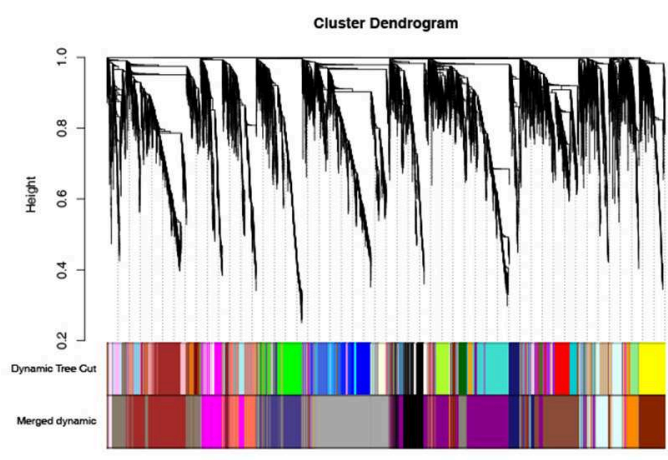

B

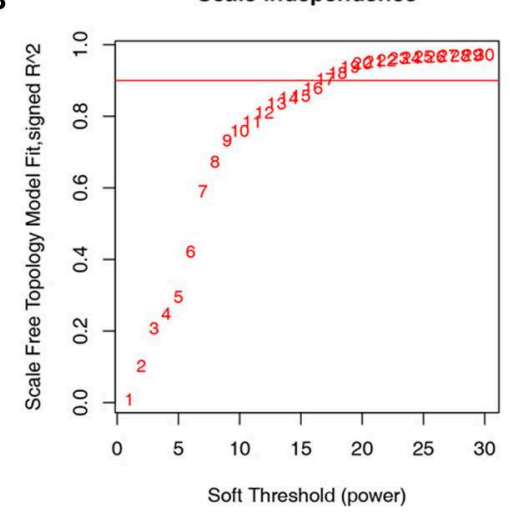

D

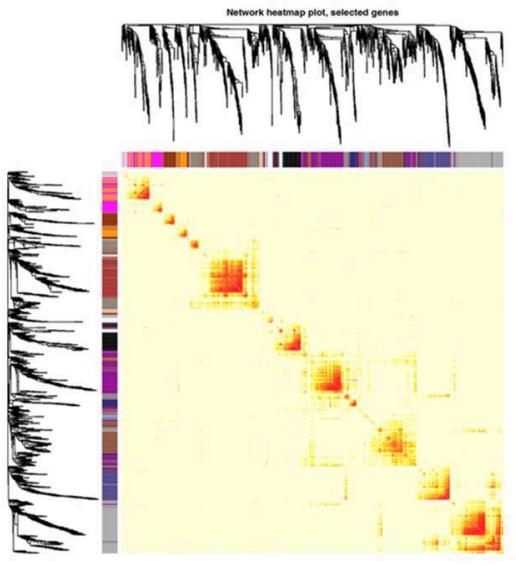

FIGURE 4 | Weighted gene co-expression network analysis (WGCNA) of genes in natural killer T-cell lymphoma (NKTCL). (A) Cluster dendrogram displays the relationship between NKTCL samples. (B) Analysis of the scale-free fit index for various soft thresholding powers ( $\beta$ ). (C) Hierarchical cluster tree showing co-expression modules identified by WGCNA. (D) Heat map plot show the topological overlap matrix (TOM) among all genes. Light color shows low overlap, and red color indicates higher overlap. The left side and the top side show the gene dendrogram and module assignment. 


\section{Identifying the Hub Genes of Natural Killer T-Cell Lymphoma}

As WGCNA generated a huge gene network, we narrowed the network by set edges weight $>0.6$ to localize the hub genes. As a result, 325 nodes and 6,900 edges were screened out for further analysis. Figure 5A showed two crucial subnetworks. The most important genes were constructed by the edges degree and kcore. The subnetwork contains mRNAs, such as LMO3, PEG3, GRB14, ASB9, and lncRNAs, such as lnc-FRG2-13, lnc-F11-2, lnc-GALNT9-4, LINC01206, probably constituting the core of the networks. To visualize these hub genes, a total of 57 genes ( $\mathrm{k}$-core $>60)$ and the top 10 core genes were listed in the volcano plots (Figure 5B). We observed the highest fold changes in LMO3 among these genes, and PEG3 is one of the most significantly changed genes. Further, the co-expression genes of LMO3 and $P E G 3$ were plotted in Figures 5C,D.

\section{Validation of Dysregulated Genes in Natural Killer T-Cell Lymphoma vs. the Normal Controls}

In order to validate the possible key genes which screened from WGCNA, we explored the top 10 genes, namely, $L M O 3$, PEG3, ASB9, GRB14, REEP1, SLC30A3, LY6K, TMEM59L, and LDHAL6B, in GEO database with the ID GSE69406, which contains three control cell line samples and five NKTCL samples. As a result, LMO3, PEG3, GRB14, and LDHAL6B were significantly upregulated in NKTCL, whereas $A S B 9$ and others displayed no significant changes between NKTCL and the controls (Figure 6A). To reveal the relationship between the expression of these genes and the survival rate, another GEO dataset with the ID GSE90597 was used to plot the Kaplan-Meier survival curve. Figures 6B-J showed the OS between the high expression group and the median/low expression group. As a

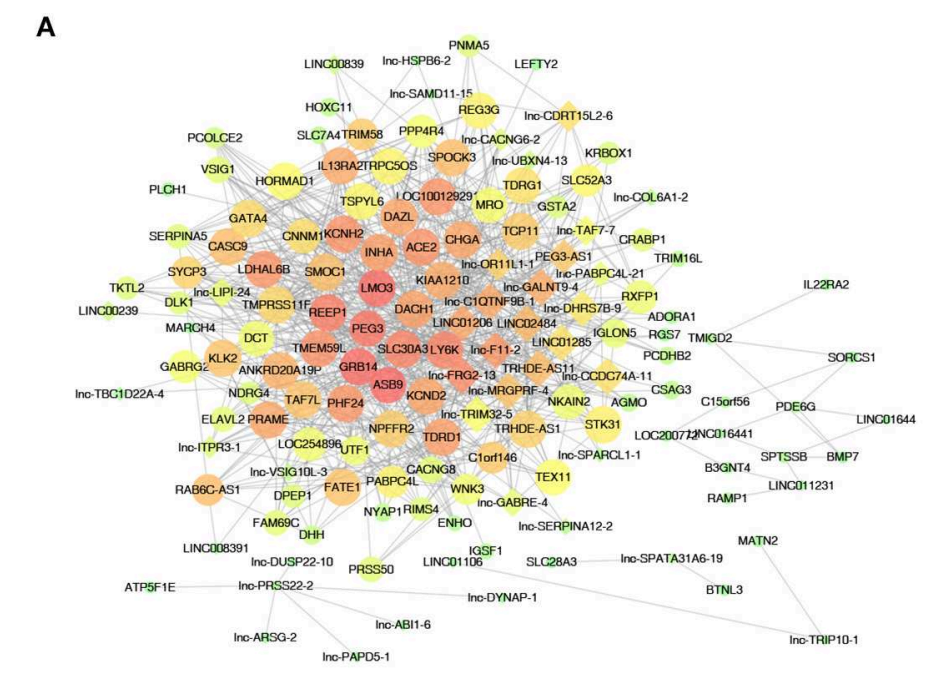

B

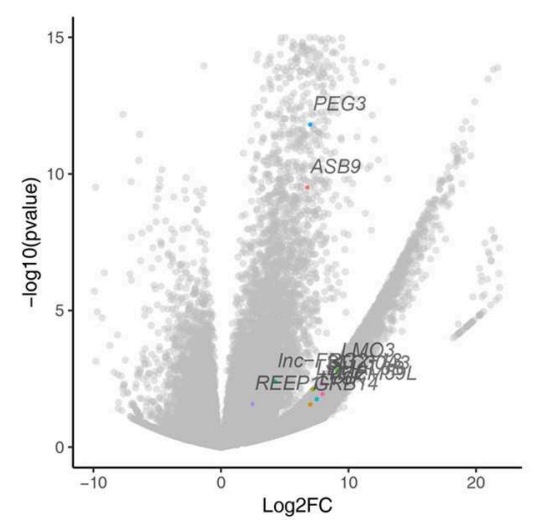

C

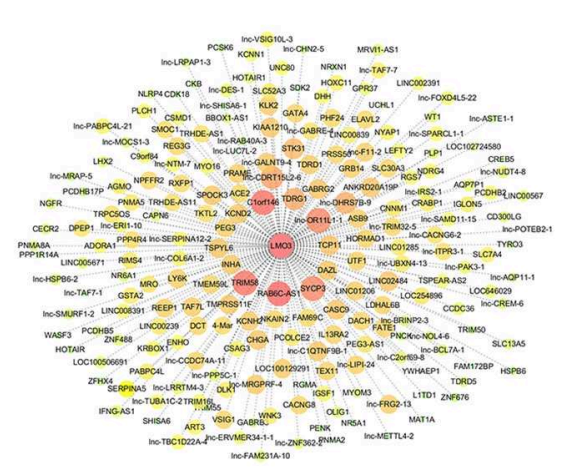

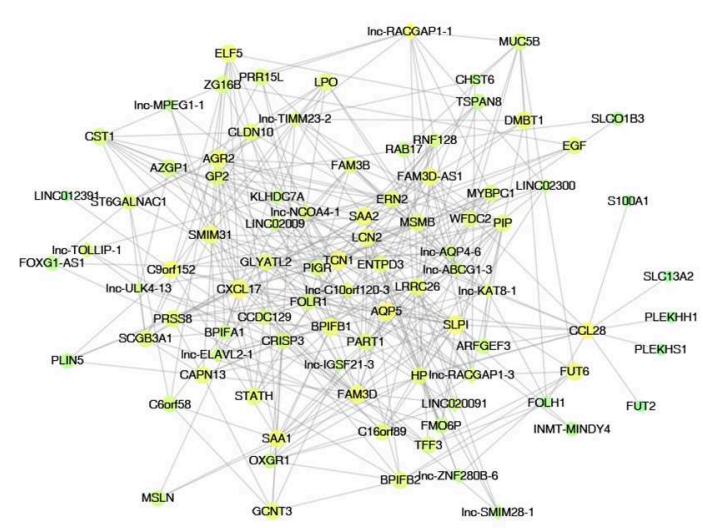

D

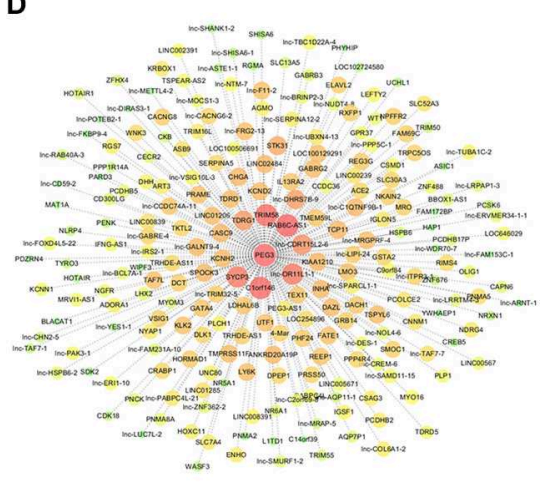

FIGURE 5 | Identified hub genes in natural killer T-cell lymphoma (NKTCL). (A) Co-expression networks constructed by weighted gene co-expression network analysis (WGCNA). Circle represents mRNA; rhombus represents long non-coding RNA; line represents correlative relationship. Large sizes and red colors represent the high degrees and k-core scoring. (B) Top 10 score genes were plot in volcano with labels and different colors. (C) Genes that co-expressed with LMO3. (D) genes that co-expressed with PEG3. 
result, the overexpression of LMO3 $(p=0.044)$ and GRB14 ( $p$ $=0.027$ ) are significantly related to the shorter period of OS.

\section{DISCUSSION}

NKTCL is biologically heterogeneous and has a variable clinical course, which most commonly affects nasal cavity and sometimes involves extra-nasal organs such as the skin, gastrointestinal tract, and testis. Since NKTCL has distinct and massive necrotic lesions, it is hard to get enough specimens to clarify the molecular mechanism of NKTCL (21). To understand the molecular mechanism in NKTCL, we utilized three datasets from the SRA database and the GEO database. We identified that LMO3 and GRB14 were the hub genes of NKTCL and found that high expressions of LMO3 and GRB14 were related to the poor prognosis. Thus, LMO3 and GRB14 might be the potential biomarkers for the diagnosis and treatment of NKTCL.

Gene expression profiling studies had provided a method to explore the molecular mechanism of NKTCL. The Homo sapiens transcriptome or gene expression dataset (SRP049695) which we used in this study was first performed by Kucuk et al. (7) to find the mutations in NKTCL. Kucuk et al. (7) found that STAT3 and STAT5B were each mutated at $5.9 \%$ frequency $(n=51)$. Baytak et al. (22) focused on the dysregulated oncogenic lncRNA (MIR155HG) in NKTCL by analyzing the SRP049695 dataset. In this study, we found that ECM receptor interaction pathway was significant in KEGG (Figure 1D), PPI network (Figure 3), and GSEA (Figure 2A) analysis.

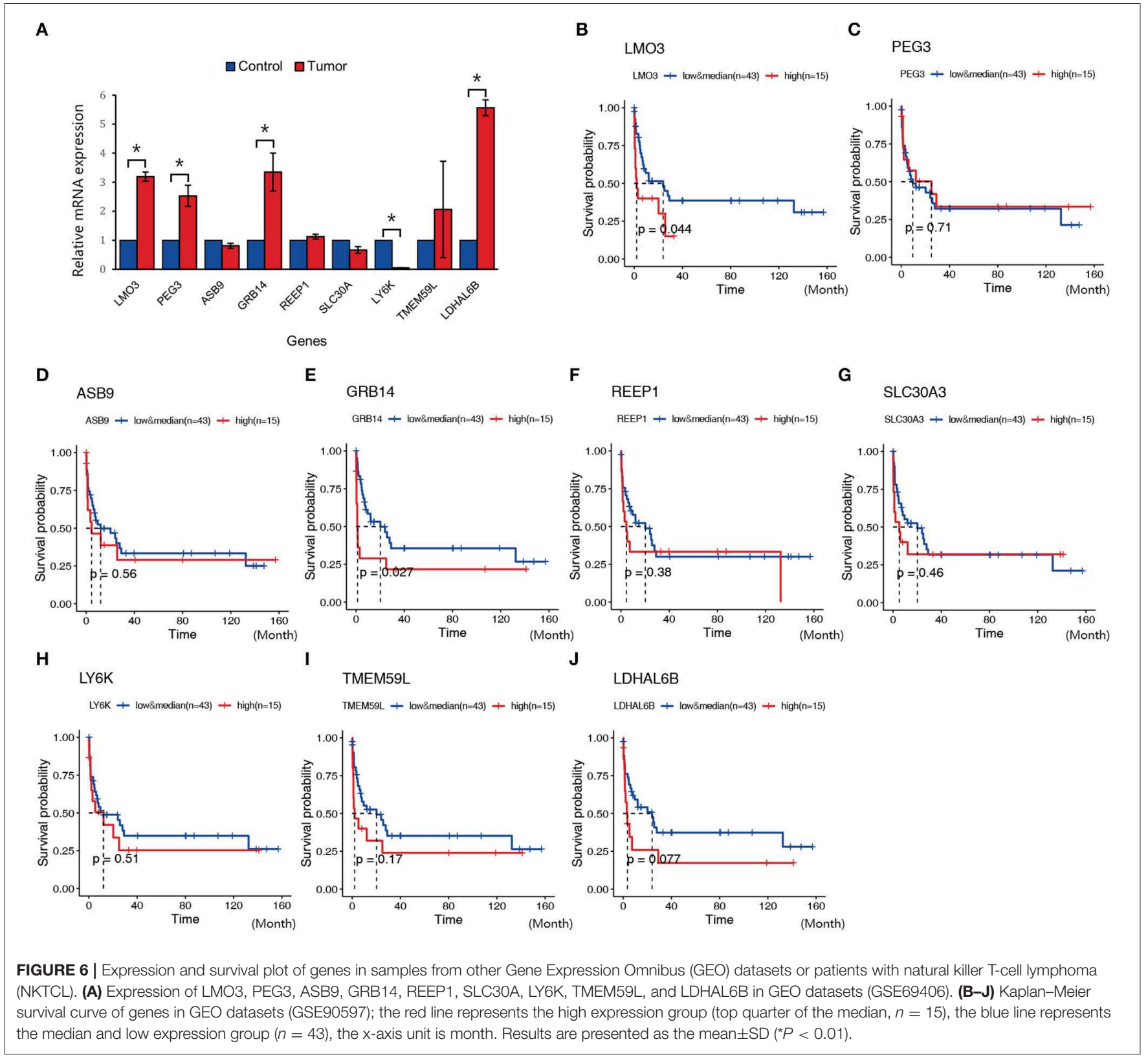


Conventional approaches for finding the core genes require a selection of hundreds of different genes, whereas WGCNA is particularly useful in summarizing many intra-modular hub genes that can be speculated for the diagnosis and prognosis of tumors $(15,23)$. In our study, LMO3 and GRB14 were screened as the hub genes of NKTCL by WGCNA. LMO3 encoded a $17 \mathrm{KD}$ protein that belongs to a cysteine-rich LIM domain protein family. The protein family also contains LMO1 and LMO2. LMO1 and LMO2 are oncogenes that are recurrently translocated and overexpressed in T-cell acute lymphoblastic leukemia (T-ALL) (24). LMO3 could promote gastric cancer cell invasion and proliferation through Akt-mTOR and AktGSK3 $\beta$ signaling pathways (25). In hepatocellular carcinoma, LMO3 directly interacts with LATS1 and suppressing Hippo signaling to promote invasion and metastasis (26). LMO3 interacts with the DNA binding domain of P53 to inhibit its activity (27). To validate the relation of LMO3 to NKTCL, another GEO dataset (GSEGSE69406) was applied in this study. It was shown that $L M O 3$ was overexpressed in tumor compared to the controls (Figure 5A). Moreover, the high expression of LMO3 was related to the low survival rate in GSE90597 dataset (Figure 5B). As a result, it is reasonable to speculate that LMO3 could be a potential oncogene and can promote the progress of NKTCL. To further validate the hypothesis of the oncogenic potency and the molecular action of LMO3 in NKTCL, further in vitro, in vivo, and clinical studies are needed and will be carried out in our future study.

GRB14 interacts with insulin receptors and shows its inhibitory effect function on receptor tyrosine kinase signaling (28). Overexpression of GRB14 facilitated STAT3 and Akt phosphorylation (29). Considering that continuous JAK-STAT pathway activation is a major feature of NKTCL, it would be very interesting to study whether GRB14 promotes the progress of NKTCL by activating STAT3 $(3,7)$. Besides, NKTCL patients have amino acid metabolism disorders, such as alanine, aspartate, and glutamate (30). Asparaginase is the most widely used antimetabolite agent in NKTCL (31). To clarify whether GRB14 is involved in the NKTCL oncogenesis by affecting metabolism has also become very meaningful. Experiments on the relationship between GRB14 and NKTCL metabolism are being conducted

\section{REFERENCES}

1. Au WY, Weisenburger DD, Intragumtornchai T, Nakamura S, Kim WS, Sng I, et al. Clinical differences between nasal and extranasal natural killer/T-cell lymphoma: a study of 136 cases from the international peripheral T-Cell Lymphoma Project. Blood. (2009) 113:3931-7. doi: 10.1182/blood-2008-10-185256

2. Vose, J. Armitage J, Weisenburger, D. International, international peripheral T-cell and natural killer/T-cell lymphoma study: pathology findings and clinical outcomes. J Clin Oncol. (2008) 26:4124-30. doi: 10.1200/JCO.2008.16.4558

3. Jiang L, Gu ZH, Yan ZX, Zhao X, Xie YY, Zhang ZG, et al. Exome sequencing identifies somatic mutations of DDX3X in natural killer/T-cell lymphoma. Nat Genet. (2015) 47:1061-6. doi: 10.1038/ng.3358.

4. Koo GC, Tan SY, Tang T, Poon SL, Allen GE, Tan L, et al. Janus kinase 3-activating mutations identified in natural killer/T-cell lymphoma. Cancer Discov. (2012) 2:591-7. doi: 10.1158/2159-8290 in our ongoing study. However, the shortcoming of this study is that it is not able to verify the hub genes in clinical samples.

In summary, we used the bioinformatics method to identify and validate hub genes of NKTCL; LMO3 and GRB14 may be the potential targets for diagnosis and treatment of NKTCL.

\section{DATA AVAILABILITY STATEMENT}

Publicly available datasets were analyzed in this study. This data can be found here: https://www.ncbi.nlm.nih.gov/sra/?term= SRP049695; https://www.ncbi.nlm.nih.gov/geo/query/acc.cgi? acc=GSE69406; https://www.ncbi.nlm.nih.gov/geo/query/acc. cgi? acc $=$ GSE90597.

\section{AUTHOR CONTRIBUTIONS}

HL, HY, and XiaL conceived and designed the study and contributed to writing of the manuscript. HL, XirL, and XiaL performed the analysis procedures. HL and ML analyzed the results. HY and ML contributed analysis tools. All authors reviewed the manuscript.

\section{FUNDING}

This study was supported by grants from the National Key Research and Development Project (2018YFC1004702), Fund of the National Natural Science Foundation of China (31970802), and Beijing Municipal Natural Science Foundation (7202099).

\section{ACKNOWLEDGMENTS}

We appreciate the SRA database (SRP049695) and GEO database (GSE69406, GSE90597) for free using.

\section{SUPPLEMENTARY MATERIAL}

The Supplementary Material for this article can be found online at: https://www.frontiersin.org/articles/10.3389/fonc. 2020.00223/full\#supplementary-material

5. Quintanilla-Martinez L, Kremer M, Keller G, Nathrath M, GamboaDominguez A, Meneses A, et al. P53 mutations in nasal natural killer/T-cell lymphoma from Mexico: association with large cell morphology and advanced disease. Am J Pathol. (2001) 159:2095-105. doi: 10.1016/S0002-9440(10)63061-1

6. Hongyo T, Hoshida Y, Nakatsuka S, Syaifudin M, Kojya S, Yang WI, et al. P53, K-ras, c-kit and BETA-catenin gene mutations in sinonasal NK/T-cell lymphoma in Korea and Japan. Oncol Rep. (2005) 13: 265-71. doi: 10.3892/or.13.2.265

7. Kucuk C, Jiang B, Hu X, Zhang W, Chan JK. Activating mutations of STAT5B and STAT3 in lymphomas derived from gammadelta$\mathrm{T}$ or NK cells. Nat Commun. (2015) 6:6025. doi: 10.1038/ ncomms 7025

8. Ng SB, Selvarajan V, Huang G, Zhou J, Feldman AL, Law M, et al. Activated oncogenic pathways and therapeutic targets in extranodal nasal-type NK/T cell lymphoma revealed by gene expression profiling. J Pathol. (2011) 223:496510. doi: $10.1002 /$ path.2823 
9. Iqbal J, Weisenburger DD, Chowdhury A, Tsai MY, Srivastava G, Greiner TC, et al. Natural killer cell lymphoma shares strikingly similar molecular features with a group of non-hepatosplenic gammadelta T-cell lymphoma and is highly sensitive to a novel aurora kinase a inhibitor in vitro. Leukemia. (2011) 25:348-58. doi: 10.1038/leu.2010.255

10. Ng SB, Yan J, Huang G, Selvarajan V, Tay JL, Lin B, et al. Dysregulated microRNAs affect pathways and targets of biologic relevance in nasal-type natural killer/T-cell lymphoma. Blood. (2011) 118:4919-29. doi: 10.1182/blood-2011-07-364224

11. Yan J, Ng SB, Tay JL, Lin B, Koh TL, Tan J, et al. EZH2 overexpression in natural killer/T-cell lymphoma confers growth advantage independently of histone methyltransferase activity. Blood. (2013) 121:4512-20. doi: 10.1182/blood-2012-08-450494

12. Selvarajan V, Osato M, Nah GSS, Yan J, Chung TH, Voon DC, et al. RUNX3 is oncogenic in natural killer/T-cell lymphomais transcriptionally regulated by MYC. Leukemia. (2017) 31:2219-27. doi: 10.1038/leu.2017.40

13. Coppo P, Gouilleux-Gruart V, Huang Y, Bouhlal H, Bouamar H, Bouchet $\mathrm{S}$, et al. STAT3 transcription factor is constitutively activated and is oncogenic in nasal-type NK/T-cell lymphoma. Leukemia. (2009) 23:166778. doi: 10.1038/leu.2009.91

14. Huang Y, de Reyniès A, de Leval L, Ghazi B, Martin-Garcia N, Travert M, et al. Gene expression profiling identifies emerging oncogenic pathways operating in extranodal NK/T-cell lymphoma, nasal type. Blood. (2010) 115:122637. doi: 10.1182/blood-2009-05-221275

15. Zhang B, Horvath S. A general framework for weighted gene coexpression network analysis. Stat Appl Genet Mol Biol. (2005) 4:17. doi: 10.2202/1544-6115.1128

16. See K, Tan WLW, Lim EH, Tiang Z, Lee LT, Li PYQ, et al. Single cardiomyocyte nuclear transcriptomes reveal a lincRNA-regulated dedifferentiation and cell cycle stress-response in vivo. Nature Commun. (2017) 8:225. doi: 10.1038/s41467-017-00319-8

17. Magani F, Bray ER, Martinez MJ, Zhao N, Copello VA, Heidman L, et al. Identification of an oncogenic network with prognostic and therapeutic value in prostate cancer. Mol Sys Biol. (2018) 14:e8202. doi: 10.15252/msb. 20188202

18. Yu G, Wang LG, Han Y, He QY. Clusterprofiler: an R package for comparing biological themes among gene clusters. OMICS. (2012) 16:2847. doi: 10.1089/omi.2011.0118

19. Subramanian A, Tamayo P, Mootha VK, Mukherjee S, Ebert BL, Gillette MA, et al. Gene set enrichment analysis: a knowledge-based approach for interpreting genome-wide expression profiles. Proc Natl Acad Sci USA. (2005) 102:15545-50. doi: 10.1073/pnas.0506580102

20. Mootha VK, Lindgren CM, Eriksson KF, Subramanian A, Sihag S, Lehar J, et al. PGC-1alpha-responsive genes involved in oxidative phosphorylation are coordinately downregulated in human diabetes. Nat Genet. (2003) 34:26773. doi: $10.1038 / n g 1180$

21. Au WY, Ma SY, Chim CS, Choy C, Loong F, Lie AK, et al. Clinicopathologic features and treatment outcome of mature T-cell and natural killercell lymphomas diagnosed according to the World Health Organization classification scheme: a single center experience of 10 years. Ann Onco. (2005) 16:206-14. doi: 10.1093/annonc/mdi037
22. Baytak E, Gong Q, Akman B, Yuan H, Chan WC, Küçük C. Whole transcriptome analysis reveals dysregulated oncogenic lncRNAs in natural killer/T-cell lymphoma and establishes MIR155HG as a target of PRDM1. Tumour Biol. (2017) 39:1010428317701648. doi: 10.1177/1010428317701648

23. Chen L, Yuan L, Qian K, Qian G, Zhu Y, Wu CL, et al. Identification of biomarkers associated with pathological stage and prognosis of clear cell renal cell carcinoma by co-expression network analysis. Front Physiol. (2018) 9:399. doi: 10.3389/fphys.2018.00399

24. Boehm T, Baer R, Lavenir I, Forster A, Waters JJ, Nacheva E, et al. The mechanism of chromosomal translocation $\mathrm{t}(11 ; 14)$ involving the T-cell receptor $\mathrm{C}$ delta locus on human chromosome $14 \mathrm{q} 11$ and a transcribed region of chromosome 11p15. The EMBO J. (1988) 7:385-94.

25. Qiu YS, Jiang NN, Zhou Y, Yu KY, Gong HY, Liao GJ. LMO3 promotes gastric cancer cell invasion and proliferation through Akt-mTOR and AktGSK3beta signaling. Int J Mol Med. (2018) 41:2755-63. doi: 10.3892/ijmm. 2018.3476

26. Cheng Y, Hou T, Ping J, Chen T, Yin B. LMO3 promotes hepatocellular carcinoma invasion, metastasis and anoikis inhibition by directly interacting with LATS1 and suppressing Hippo signaling. J Exp Clin Cancer Res. (2018) 37:228. doi: 10.1186/s13046-018-0903-3

27. Larsen S, Yokochi T, Isogai E, Nakamura Y, Ozaki T, Nakagawara A. LMO3 interacts with p53 and inhibits its transcriptional activity. Biochem Biophys Res Commun. (2010) 392:252-7. doi: 10.1016/j.bbrc.2009.12.010

28. Béréziat V, Kasus-Jacobi A, Perdereau D, Cariou B, Girard J, Burnol AF. Inhibition of insulin receptor catalytic activity by the molecular adapter Grb14. J Biol Chem. (2002) 277:4845-52. doi: 10.1074/jbc.M1065 74200

29. Balogh K, Asa SL, Zheng L, Cassol C, Cheng S, Ezzat S. The insulin resistance Grb14 adaptor protein promotes thyroid cancer ret signaling and progression. Oncogene. (2012) 31:4012-21. doi: 10.1038/onc. 2011.569

30. Xu PP, Xiong J, Cheng S, Zhao X, Wang CF, Cai G, et al. A phase II study of methotrexate, etoposide, dexamethasone and pegaspargase sandwiched with radiotherapy in the treatment of newly diagnosed, stage IE to IIE extranodal natural-killer/T-cell lymphoma, nasal-type. EBioMed. (2017) 25:41-9. doi: 10.1016/j.ebiom.2017.10.011

31. Campalani E, Arenas M, Marinaki AM, Lewis CM, Barker JN, Smith CH. Polymorphisms in folate, pyrimidine, and purine metabolism are associated with efficacy and toxicity of methotrexate in psoriasis. J Invest Dermatol. (2007) 127:1860. doi: 10.1038/sj.jid.5700808

Conflict of Interest: The authors declare that the research was conducted in the absence of any commercial or financial relationships that could be construed as a potential conflict of interest.

Copyright (C) $2020 \mathrm{Liu}, \mathrm{Liu}, \mathrm{You}, \mathrm{Li}$ and Li. This is an open-access article distributed under the terms of the Creative Commons Attribution License (CC BY). The use, distribution or reproduction in other forums is permitted, provided the original author(s) and the copyright owner(s) are credited and that the original publication in this journal is cited, in accordance with accepted academic practice. No use, distribution or reproduction is permitted which does not comply with these terms. 